\title{
Effect of Annealing Temperature on the Magnetic Properties of Pr-Fe-Co-Zr-Nb-B Alloys Ribbons
}

\begin{abstract}
K. PAWLIK*
Institute of Physics, Częstochowa University of Technology, Al. Armii Krajowej 19, 42-200 Częstochowa, Poland

The properties of rapidly solidified ribbons of $\mathrm{Pr}_{9} \mathrm{Fe}_{50+x} \mathrm{Co}_{13} \mathrm{Zr}_{1} \mathrm{Nb}_{4} \mathrm{~B}_{23-x}$ (where $x=2,5,8$ ) alloys annealed at temperatures ranging from $923 \mathrm{~K}$ to $1033 \mathrm{~K}$ were investigated. The heat treatment of the fully amorphous as-cast ribbons led to the simultaneous crystallization of the hard magnetic $\operatorname{Pr}_{2}(\mathrm{Fe}, \mathrm{Co})_{14} \mathrm{~B}$, the soft magnetic $\alpha$-Fe and the paramagnetic $\operatorname{Pr}_{1+x} \mathrm{Fe}_{4} \mathrm{~B}_{4}$ phases. However, the crystallization of the $\alpha$-Fe phase took place as the second crystallization event at higher temperatures for the $x=8$ alloy. The Rietveld analysis of the X-ray diffraction patterns was used to calculate the crystallite sizes of constituent phases. Additionally, using PONKCS method the weight fractions of crystalline and amorphous components were quantified. For all alloy compositions the temperature dependencies of magnetic parameters were determined from the hysteresis loops.
\end{abstract}

DOI: 10.12693/APhysPolA.133.657

PACS/topics: 75.50.Ww, 75.50.Tt, 81.40.Ef

\section{Introduction}

Previous studies were focused on determining the glass forming abilities of the $\mathrm{Pr}_{9} \mathrm{Fe}_{50+x} \mathrm{Co}_{13} \mathrm{Zr}_{1} \mathrm{Nb}_{4} \mathrm{~B}_{23-x}$ (where $x=0,2,5,8$ ) alloys [1] and the phase formation during annealing of the rapidly solidified ribbons. It was shown that all investigated alloys reveal large supercooled liquid region before crystallization $\Delta T_{x}$ reaching up to $112 \mathrm{~K}$ for the amorphous $\operatorname{Pr}_{9} \mathrm{Fe}_{58} \mathrm{Co}_{13} \mathrm{Zr}_{1} \mathrm{Nb}_{4} \mathrm{~B}_{15}$ alloy ribbon. In [2] detailed studies of the microstructure, phase constitution and magnetic properties of these alloy ribbons subjected to annealing at various temperatures were presented. Furthermore, the magnetic interactions between grains of constituent phases were analysed. In the present work the research was focused on comparison of the magnetic properties and formation of crystalline phases during annealing of ribbons of the $\mathrm{Pr}_{9} \mathrm{Fe}_{50+x} \mathrm{Co}_{13} \mathrm{Zr}_{1} \mathrm{Nb}_{4} \mathrm{~B}_{23-x}(x=2,5,8)$ alloys.

\section{Samples preparation and experimental methods}

The samples were obtained in the form of melt-spun ribbons of the nominal compositions $\mathrm{Pr}_{9} \mathrm{Fe}_{50+x} \mathrm{Co}_{13} \mathrm{Zr}_{1} \mathrm{Nb}_{4} \mathrm{~B}_{23-x} \quad(x=2, \quad 5,8)$. The specimens were sealed off in the quartz tubes in the argon atmosphere and annealed at temperatures ranging from $923 \mathrm{~K}$ to $1033 \mathrm{~K}$ for $5 \mathrm{~min}$. The phase constitution was analyzed using Rietveld refinement of the X-ray diffraction spectra. The diffraction patterns were collected using Bruker D8 Advance diffractometer operating in the Bragg-Brentano configuration. The diffractometer was equipped with $\mathrm{Cu} \mathrm{X}$-ray tube and the $\mathrm{K}_{\beta}$ filter on detector side. The instrument was also equipped with Soller slits on incident and diffracted

\footnotetext{
*e-mail: kpawlik@wip.pcz.pl
}

beam, the LynxEye detector with linear focus of 25 $\mathrm{mm}$ and incident beam divergence slit of $0.6 \mathrm{~mm}$. The measurements were performed with the $2 \theta$ step size of 0.02 deg. and step time $5 \mathrm{~s}$. In order to quantify the amorphous and crystalline phases the PONKCS (Partial Or No Known Crystal Structure) method was applied [3]. The crystallite sizes were calculated taking into account the instrument contribution to the diffraction lines broadening. This contribution was determined by the Rietveld analysis of the diffraction spectrum measured for the $\mathrm{LaB}_{6}$ standard sample. The hysteresis loops were measured using the LakeShore 7307 VSM magnetometer operating in the external magnetic fields up to $2 \mathrm{~T}$ at room temperature.

\section{Results and discussion}

The samples of all alloy compositions were fully amorphous in the as-cast state and their corresponding hysteresis loops were typical for the soft magnetic materials. The saturation polarization $\left(J_{s}\right)$ of about $0.87 \mathrm{~T}$ was similar for all alloy compositions. Most of the samples annealed at various temperatures $\left(T_{a}\right)$ ranging from $923 \mathrm{~K}$ to $1033 \mathrm{~K}$ exhibited hard magnetic properties. The selected hysteresis loops measured for ribbons of all compositions annealed at $923 \mathrm{~K}$ are shown in Fig. 1. The hysteresis loop for the $x=2$ alloy ribbon annealed at $923 \mathrm{~K}$ has characteristic wasp-waisted shape. This indicates that $T_{a}=923 \mathrm{~K}$ was not sufficient to drive the phase evolution to the point were the hard magnetic properties are achieved.

The dependences of magnetic parameters on the annealing temperature $T_{a}$ determined for all investigated ribbons are presented in Fig. 2. For the $x=5$ and 8 alloys the $J_{s}$ increases with the increase of annealing temperature. This effect is more pronounced for the $x=5$ alloy sample where the saturation polarization rises from $0.69 \mathrm{~T}$ for $T_{a}=923 \mathrm{~K}$ to $0.89 \mathrm{~T}$ for $T_{a}=1033 \mathrm{~K}$. For the $x=2$ alloy annealed at $923 \mathrm{~K}$ the $J_{s}$ reaches the highest value of $0.88 \mathrm{~T}$, while for higher $T_{a}$ the $J_{s}$ decreases to 


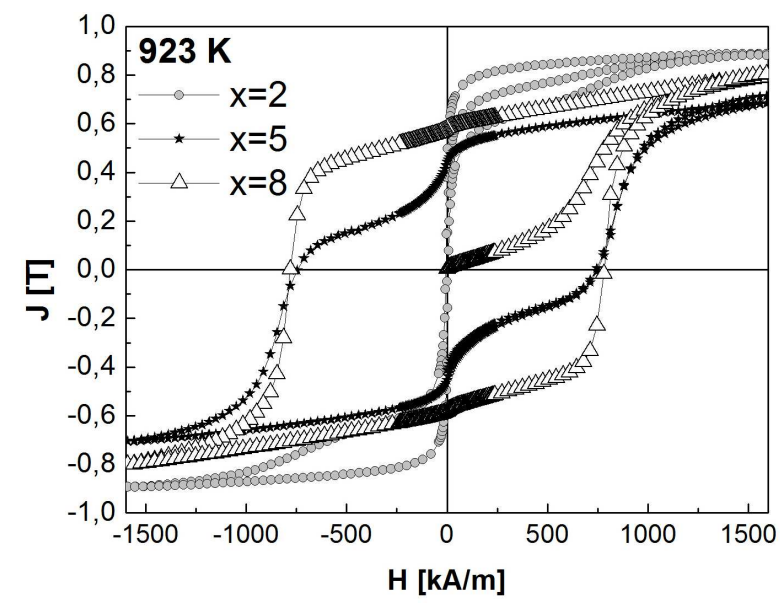

Fig. 1. The hysteresis loops measured for ribbons of $x=2,5,8$ alloys annealed at $923 \mathrm{~K}$.

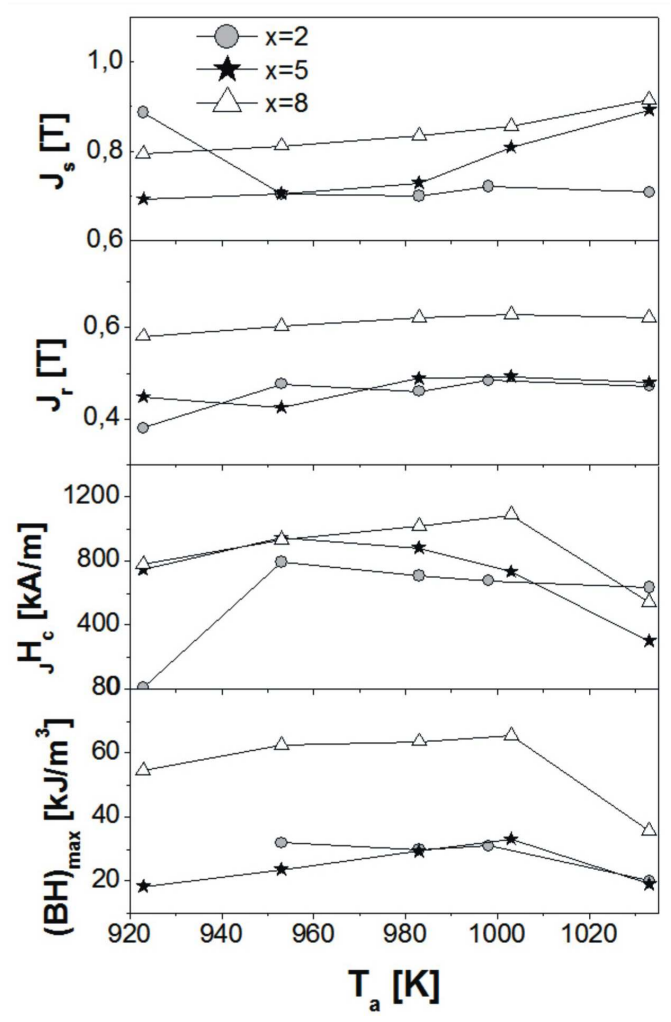

Fig. 2. The dependences of magnetic parameters on the annealing temperature $T_{a}$ for ribbons of the $x=2$, 5,8 alloys.

0.7 $\mathrm{T}$ and remains almost independent on the annealing temperature.

The remanence $\left(J_{r}\right)$ seams to depend on the alloy composition and reaches the highest values for the $x=8$ alloy. Furthermore, the annealing conditions do not influence significantly the remanence for all alloys. In particular for the $x=8$ alloy ribbons the remanence increases only by $\sim 8 \%$ when annealed at $1003 \mathrm{~K}$ in respect to $J_{r}$ measured for ribbon annealed at $T_{a}=923 \mathrm{~K}$. All the annealed samples (except for $x=2$ alloy, $T_{a}=923 \mathrm{~K}$ ) ex- hibit the remanence enhancement. The highest $J_{r} / J_{s}=$ 0.74 was determined for $x=8$ alloy annealed at 983 and $1003 \mathrm{~K}$

The effect annealing temperature is more pronounced in case of the coercivity $\left({ }_{J} H_{c}\right)$. For $x=2$ and 5 alloys the ${ }_{J} H_{c}$ reaches the maximum values after the annealing at $953 \mathrm{~K}$ and then decreases for higher $T_{a}$. In case of the $x=8$ alloy the highest coercivity is reached for $T_{a}=$ $1003 \mathrm{~K}$. The maximum energy product $(B H)_{\max }$ depends strongly on the remanence values and is the highest for the $x=8$ alloy, reaching $65 \mathrm{~kJ} / \mathrm{m}^{3}$ for $T_{a}=1003 \mathrm{~K}$.

It is of great importance to identify and quantify the constituent phases of the alloys subjected to annealing in order to understand the temperature dependences of the magnetic parameters. The XRD studies shown that the annealed samples consist of three main crystalline phases: the paramagnetic $\operatorname{Pr}_{1+\varepsilon} \mathrm{Fe}_{4} \mathrm{~B}_{4}$, the hard magnetic $\operatorname{Pr}_{2}(\mathrm{Fe}, \mathrm{Co}){ }_{14} \mathrm{~B}$ and the soft magnetic $\alpha$-Fe. A very small amount of $\mathrm{Pr}_{2} \mathrm{O}_{3}$ oxide for the highest annealing temperature was also detected. Additionally, depending on the annealing temperature and the composition of the alloy a presence of the amorphous phase was also possible, but not obvious from visual inspection of the diffraction patterns. In order to determine the fractions of constituent phases formed during annealing the Rietveld refinement of the spectra was performed. Due to the fact that in traditional Rietveld analysis quantification of amorphous phase is impossible, the technique known as PONKCS [3] was used. The example of refined pattern with corresponding difference curve is presented in Fig. 3.

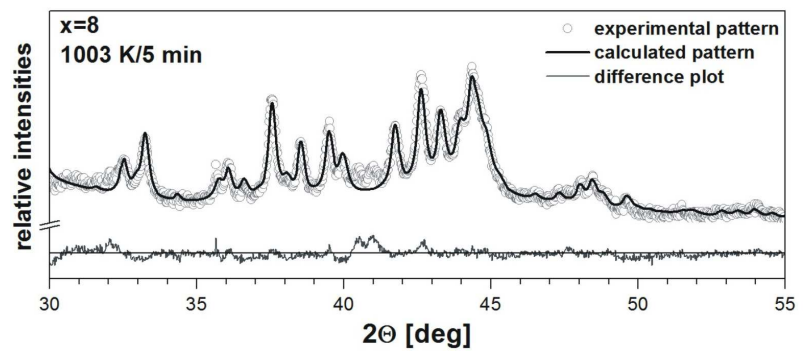

Fig. 3. The experimental and calculated diffraction pattern with corresponding difference curve for $\mathrm{Pr}_{9} \mathrm{Fe}_{58} \mathrm{Co}_{13} \mathrm{Zr}_{1} \mathrm{Nb}_{4} \mathrm{~B}_{15}$ alloy ribbons annealed at $1003 \mathrm{~K}$.

Additionally the crystallite sizes of constituent phases were calculated as the volume weighted coherently diffracting domain sizes. The dependences of the crystallite sizes and weight fractions of constituent phases on the annealing temperature are presented in Fig. 4 for all alloys compositions.

The $x=2$ alloy ribbons annealed at $923 \mathrm{~K}$ contain about 60 wt. $\%$ of amorphous phase. It was estimated by Kneller et al. in [4] for nanocomposite hard magnetic alloys, that the amount of hard magnetic phase sufficient to attain high coercivity and provide remanence enhancement is about 9 vol.\%. However, in case of studied alloys low weight fraction of hard magnetic 2:14:1 phase 

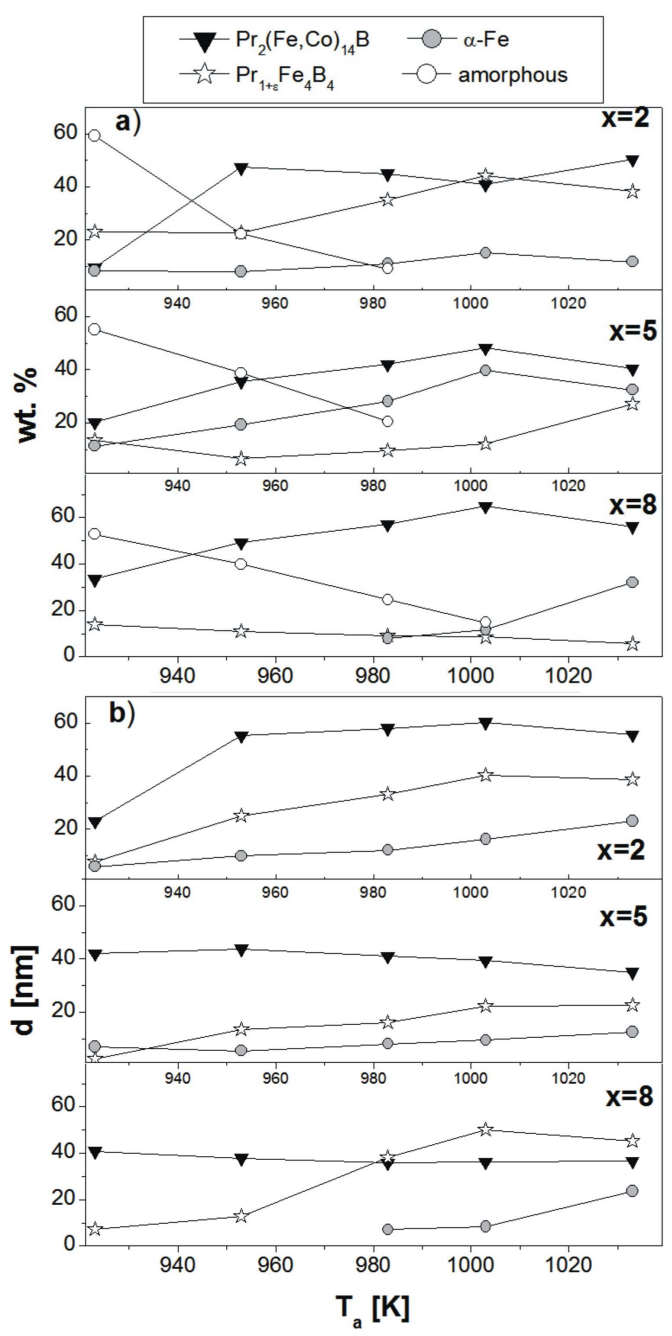

Fig. 4. The dependences of the weight fractions of constituent phases (a) and their crystallite sizes (b) on the annealing temperature $T_{a}$ for $\mathrm{Pr}_{9} \mathrm{Fe}_{50+x} \mathrm{Co}_{13} \mathrm{Zr}_{1} \mathrm{Nb}_{4} \mathrm{~B}_{23-x}$ alloys (where $x=2,5,8$ ).

$(\sim 9.6 \%)$ is too small to achieve nanocomposite material with good hard magnetic properties. The amount of hard magnetic phase that crystallizes during annealing at $923 \mathrm{~K}$ depends on the alloy composition. For $x=5$ and $x=8$ alloys the weight fractions of $2: 14: 1$ phase are $\sim 20$ and $33 \%$, respectively. With the increase of the fraction of this phase the evolution of shapes of the hysteresis loops was observed (Fig. 1.). For the $x=8$ alloy ribbon, annealed at $923 \mathrm{~K}$, the 33 wt. $\%$ of $2: 14: 1$ phase and 52 wt. $\%$ of soft magnetic amorphous phase yield the remanence enhancement and high coercivity.

With increase of the annealing temperature the amount of the amorphous phase decreases and the weight fractions of the crystalline components rise. The samples with the highest energy product (for $x=8$ alloy ribbons, annealed at 983 and $1003 \mathrm{~K}$ ) consist of $\sim 57-65 \mathrm{wt} . \%$ of hard magnetic phase and $\sim 32-26$ wt. $\%$ of soft magnetic phases (the amorphous and $\alpha$-Fe phases).

The grain sizes of the crystalline phases have major impact on the coercivity of the nanocomposite material as well as on the remanence enhancement effect $[4,5]$. For all investigated ribbons the crystallite sizes of the hard magnetic phase $\mathrm{Pr}_{2}(\mathrm{Fe}, \mathrm{Co}){ }_{14} \mathrm{~B}$ remain almost constant (of $\sim 40 \mathrm{~nm}$ ) for all annealing temperatures. However, for the $x=2$ ribbon annealed at $923 \mathrm{~K}$ the mean crystallite size of the 2:14:1 phase is about $20 \mathrm{~nm}$. This is probably related to the high temperature stability of the amorphous phase for this alloy composition as was reported in [1]. The crystallite sizes of the paramagnetic phase increase with the annealing temperature from about $10 \mathrm{~nm}$ dia. for $923 \mathrm{~K}$ to about $40-50 \mathrm{~nm}$ for 1003 K. Furthermore, annealing of ribbons at $923 \mathrm{~K}$ resulted in precipitation of very fine crystallites $(\sim 8 \mathrm{~nm}$ dia.) of the $\alpha$-Fe phase for $x=2$ and 5 alloys. In case of the $x=8$ alloy ribbons the $\alpha$-Fe crystallizes at $983 \mathrm{~K}$. Further increase of $T_{a}$ resulted in growth of crystals and increase of weight fraction of $\alpha$-Fe phase for all studied compositions. This has a detrimental effect on the coercivity and maximum energy product of the samples. The calculated crystallite sizes of constituent phases are in good agreement with those obtained from the TEM studies [2].

\section{Conclusions}

The Rietveld analysis of diffraction patterns using PONKCS method allowed to determine the phase constitution changes during annealing of ribbons of the $\mathrm{Pr}_{9} \mathrm{Fe}_{50+x} \mathrm{Co}_{13} \mathrm{Zr}_{1} \mathrm{Nb}_{4} \mathrm{~B}_{23-x}(x=2,5,8)$ alloys as well as to calculate the crystallite sizes of precipitating phases. The increase of annealing temperature resulted in the reduction of the content of the amorphous phase accompanied by the increase of weight fraction of the hard magnetic 2:14:1 and soft magnetic $\alpha$-Fe phases. The weight fraction of crystalline phases is strictly controlled by the chemical composition of the alloy. Especially, the amount of the paramagnetic $\operatorname{Pr}_{1+x} \mathrm{Fe}_{4} \mathrm{~B}_{4}$ phase decreases with the increase of Fe content. Furthermore, the $\mathrm{Fe} / \mathrm{B}$ ratio has an effect in the thermal stability of the amorphous phase. As a consequence, both factors have the influence on the magnetic properties of the alloys. The grain sizes of the 2:14:1 phase remain almost unchanged with the annealing temperature. However, in case of the $\alpha$-Fe phase the grain size increases with $T_{a}$ for all alloy compositions. This effect leads to decrease of ${ }_{J} H_{c}$ and $(B H)_{\max }$ as well as change of the demagnetization curves. The best magnetic properties were measured for the $x=8$ alloy ribbons annealed at $1003 \mathrm{~K}$.

\section{References}

[1] K. Pawlik, P. Pawlik, W. Kaszuwara, J.J. Wysłocki, Acta Phys. Pol. A 131, 979 (2017).

[2] K. Pawlik, Acta Phys. Pol. A 131, 1264 (2017).

[3] N.V.Y. Scarlett, I.C. Madsen, Powder Diffr. 21, 278 (2006).

[4] E.F. Kneller, R. Hawig, IEEE Trans. Magn. 273588 (1991).

[5] R. Skomski, J.M.D. Coey, Phys. Rev. B 4815812 (1993). 